\title{
Use of a Smart Phone based Application to Measure Roughness of Polyurethane Stabilized Concrete Pavement
}

\author{
Fortunatus $\mathbf{M}^{*}$, Onyango M, Fomunung I, McLean A and Owino J \\ University of Tennessee at Chattanooga (UTC), USA
}

Submission: February 21, 2018; Published: April 26, 2018

*Corresponding author: Fortunatus M, Department of Civil Engineering, University of Tennessee at Chattanooga (UTC), USA,

Email: Mawazo-Fortunatus@mocs.utc.edu

\begin{abstract}
To evaluate the long-term performance of polyurethane treated concrete pavement sections, indicators such as ride quality, surface elevation, surface deflection, and subbase/subgrade structural integrity are usually assessed by using different tools. This paper presents the evaluation of ride quality of polyurethane stabilized sections of the United States interstate I-24 and I-75 in Chattanooga, Tennessee with lengths ranging from $1050 \mathrm{~m}(0.65 \mathrm{mi}$.) to $3220 \mathrm{~m}$ ( $2.0 \mathrm{mi}$.) by using two instruments; (1) a standard inertial profiler, and (2) an android smartphone-based application called Roadroid. The analysis of raw data from the profiler were conducted using a profile viewing and analyzing software (ProVAL) to obtain the international roughness index (IRI) - a worldwide ride quality measure. Roadroid IRI values were repeatable with some deviations which are due to variation of lead in lengths, tire wander, and/or minor over-correction of the treated slabs where by adjacent slabs are not at the same elevation. Comparison of the IRI values from ProVAL software and those collected using the smartphone app yields a linear correlation. However, results show that a smartphone app underestimates IRI measurements, and the differences are statistically significant
\end{abstract}

Keywords: Smartphone; Polyurethane; Slab stabilization; International roughness index; Ride quality

Abbreviations: FHWA: Federal Highway Administration; DOT: Department of Transportation; NCHRP: National Cooperative Highway Research Program; TRB: Transportation Research Board; TDOT: Tennessee Department of Transportation; MRI: Mean Roughness Index; CV: Coefficient of Variation

\section{Background}

The Federal Highway Administration (FHWA) categorizes rigid (concrete) pavement rehabilitation and preservation treatments technique in the following groups; joint resealing, slab stabilization (pressure grouting), partial/full depth repairs, load transfer restoration, and diamond grinding and grooving [1].

Polyurethane injection is one among the slab stabilization/ pressure grouting method, others being cement-flyash grouts and asphalt grouts. The main purpose of slab stabilization is to fill the voids of a supporting layer, therefore reducing both deflection and its related distresses such as pumping and faulting [1].

Polyurethane injection emerged in recent years as a concrete pavement treatment technique, and some State Department of Transportations (DOTs) have shown interest in adopting it as one of their pavement management technique. Different factors such as roughness, difference in elevations among the adjacent slabs, permeability and structural integrity of the subbase/ subgrade are assessed to project the long-term performance of the pavement section treated with polyurethane.

To examine the performance of a $9.70 \mathrm{~km}(6.03 \mathrm{mi}$.) sectionfour lane divided highway, treated by polyurethane injection under the Pennsylvania DOT the following indicators were studied:

i. Changes in surface elevation - using arobotic total station, ii. Strength of the subbase-using a dynamic cone penetrometer,

iii. Surface deflection of the slabs - using a falling weight deflectometer and

iv. Ride quality (measured in IRI) - using high speed profiler [2].

Ride quality is among the four indicators used to evaluate the pavement rehabilitation and treatment needs, others being pavement distress, pavement structural integrity and skid 
resistance. It is evaluated by measuring the roughness of the pavement in IRI- a World Bank index which is based on a quarter car simulation model [3].

Pavement roughness is the longitudinal unevenness of the road profile that affects the road-vehicle interactions, and therefore the comfortability and safety of road users. It is computed from different methods ranging from traditional (subjective) rating methods such as pavement condition rating to more modern and objective precise methods of using a laser based inertial referencing system (profilers) in which an accelerometer is used to create an inertial plane of referencing and vehicle mounted lasers are used to measure the deviation of longitudinal profile from that plane.

In recent years, smartphone applications evolved as a quick IRI measuring tools. Roadroid, among others, has emerged as a roughness measuring tool, based on the dynamic response of the vehicle as it travels on the road at a specified speed.

Like other smartphones apps, Roadroid has an advantage of being less costly, simple and portable as compared to other roughness measuring instruments. It needs a maximum of two personnel who drive and collect data over the section to be surveyed, and then the raw data is uploaded to the developer database for analysis.

This paper evaluates the effectiveness of Roadroid app as one of the tools for monitoring and assessing the ride quality of polyurethane stabilized concrete pavement sections if they are within the expected condition or requiring further treatment(s).

\section{Road Surface Roughness}

Road roughness is a phenomenon that results from the interaction of the road profile and the vehicle moving along the road. Road roughness is affected by parameters such as vehicle's suspension (includes how the tires are connected to the vehicle body with springs and shock absorber), tire pressure and human body sensibility to vibration as the vehicle travels at a certain speed [3].

Road comfort and safety to its users is mostly related to smoothness (low roughness) of the road surface. Road roughness also affects vehicle operating costs (cost of the tires, fuels, vehicle maintenance and repairs cost).

IRI was agreed as an index for measuring the road roughness after the international road roughness experiment which was conducted in Brasilia, Brazil in 1982 [3]. IRI is derived from the road longitudinal profile, which is the measure of road elevation in the wheel track encountered by the vehicle as it moves [4].

Instruments and tests are used to produce a sequence of numbers related to a "true profile" for an imaginary line of the road. Sometimes the true profile is not obtained, instead its components are used for the analysis [5].
Static and automated methods are available for measuring pavement profile. The static methods are slower, time consuming and liable to human errors. The mentioned drawbacks of static methods make them less preferred over the dynamic methods (automated instruments) which are faster and give profiles with high accuracy due to less likelihood of human error. Automated roughness measuring instruments such as inertial profilers are costly to operate. In response to the cost of roughness data collection for a questionnaire on automated distress collection techniques conducted by the Transportation Research Board (TRB) team as part of the National Cooperative Highway Research Program (NCHRP), two agencies reported the cost to vary from $\$ 2.23$ per mile to $\$ 10.00$ per mile with an average cost of $\$ 6.12$ per mile [6].

Roughness measuring instruments are classified based on their precision and accurateness of measuring the true profiles. Listed are the four classification of roughness measuring instruments [3,7-9]:

- $\quad$ Class 1: Precision profiles

- $\quad$ Class 2: Other profilometric method

- $\quad$ Class 3: IRI estimates from correlation equations

- $\quad$ Class 4: Subjective ratings and uncalibrated measures

Profile measurements must be continuous or closely spaced to capture the influence on distortions (pavement roughness) on ride quality. Road roughness is mainly measured by either of the two measuring systems:

i. Profilometers/profilers which use the principle of inertial profilometry to measure the longitudinal profiles and convert them into a roughness index, and

ii. Response Type Road Roughness Measuring Systems (RTRRMS) which measure the response of the vehicle to the longitudinal profiler $[7,10]$.

\section{Inertial profilometers}

Inertial profilometers are contactless with the road and their measure is not based on the response of the vehicle to the road. They are the higher speed road profiling systems, if they are operated within the limitation of their wavelength and amplitude, the profile measurement is absolute (i.e. no need to compare with other profile measuring system). Inertial profilometers have the following components: height sensor, accelerometer, odometer, and a processing device/computer $[7,11]$.

The accelerometer measures the vertical acceleration of the vehicle as it traverses the pavement, the accelerations are integrated twice to quantify the vertical displacement of the vehicle body. This displacement is added to distance from the height sensor to obtain the pavement profile elevation [7]. 


\section{Civil Engineering Research Journal}

After collecting the profiles on a pavement using profilers, digital readings are sent to a computer where a software developed or specified by the manufacturer will be used for analysis. For most profilers in practice profiles are collected in a sample interval ranging from $25 \mathrm{~mm}$ to $360 \mathrm{~mm}$ [4].

\section{Response type road roughness measuring systems}

They measure the dynamic response of the vehicles to the road longitudinal profile either mechanically or by using accelerometers. RTRRMS has a drawback of requiring regular calibration since each vehicle has its own suspension system that changes over time [10].

\section{Evaluation of road roughness by smartphones}

Using the tri-axial accelerometer embedded in Samsung galaxy with android operating System 2.4, a team at the University of Illinois at Urbana-Champaign researched on the possibility of collecting roughness measurements using the smartphone. Findings from this study are;

i. $\quad$ For road section with low to medium roughness IRI values from the standard inertial profiler and the smartphone are the same with few outliers,

ii. The smartphone application underestimated roughness measurements for road section with relatively high roughness and

iii. IRI measurements collected using the smartphone application were repeatable and acceptable for the intended application [12].

Another similar study was done by Hanson, Cameron and Hildebrand [13] to evaluated the possibility of using low cost consumer level mobile devices on measuring road roughness; eleven (11) test scenarios were conducted on a $1 \mathrm{~km}(0.62 \mathrm{mi})$ segment of a secondary road in New Brunswick, Canada where three smartphone brands (iPhone 5, Galaxy SIII and Blackberry Z10) with the tri-axial accelerometer embedded in them were mounted either on the windshield, vent or T-bracket of or in a 2001 Pontiac Sunfire (compact car), sports utility vehicle (SUV) - 2011 Nissan Rogue or a 2008 Ford F250 truck. Results from this study shows that IRI values measured by the inertial profiler were slightly higher compared to those from the smartphones [13].

The evolution of smartphones in 2010 motivated the Roadroid team, to review and improve their drawbacks of since 2002, and developed an android based application called Roadroid that evaluates pavement smoothness based on an in-built smartphone tri-axial accelerometer. Roadroid has advantages of low cost, portability and ease of use as compared to other instruments $[8,14]$. After conducting the survey, the operator uploads the raw data to the manufacturer database, and then logs in to his/her account to obtain the IRI measurements. The analyzed data can be aggregated in different segments depending on the section length and sample size required for further analysis by the operator.

Roadroid has also a capability of capturing geographic positioning systems photos during the survey, along with the collected data, the photos can be processed with their respective location, or used for in-office visual inspection of the road surface in case there are other pavement distresses to be assessed.

Roadroid collects roughness data at class 3 level (based on correlation equations), it is very easy and cost effective compared to class 2 instruments, also useful in roads where expensive and complex instruments cannot be used (that is bicycle roads and in developing countries). While compared to class 4 level (subjective rating), Roadroid is objective, highly portable and gives powerful and effective data collection and visualization on internet map [15].

Roadroid is a response type road roughness measuring system; therefore, the IRI will vary depending on the vehicle type, operating speed of the vehicle and road surface condition. A research done in 2013 by the University of Pretoria found that estimated IRI obtained from Roadroid yielded consistent results if factors such as speed, pavement surface, tire pressure etc. are standardized [8]. Roadroid gives two options for obtaining the roadway section IRI $[8,15]$ :

- $\quad$ Estimated IRI (eIRI) which is based on the peak and root mean square vibration analysis. The eIRI values are collected at a speed of $20-100 \mathrm{~km} / \mathrm{hr}$.

- $\quad$ Calculated IRI (cIRI) which is based on the quarter car simulation. Data are collected at a speed of $60-80 \mathrm{~km} / \mathrm{hr}$. The operator can set a known reference when measuring cIRI.

Roadroid can also be used in winter to determine the performance of snow-removal and ice grinding. Moreover, in frost and heave actions it tells when and where it is happening by comparing the situation to the previous event IRI [8]. Roadroid classifies ride quality in IRI according to Table 1.

Table 1: Roadroid Ride Quality Scale.

\begin{tabular}{|c|c|c|}
\hline IRI (m/km) & Speed (km/hr.) & Ride Quality \\
\hline$<2.2$ & $>70$ & Good \\
\hline $2.2-3.8$ & $50-70$ & Ok \\
\hline $3.8-5.4$ & $30-50$ & Fair \\
\hline$>5.4$ & $<30$ & Poor \\
\hline
\end{tabular}

\section{Data Collection}

A contractor hired by the Tennessee Department of Transportation (TDOT) collected the raw profile data using a standard inertial profiler. Profiler runs were conducted on four sections with lengths ranging from $1050 \mathrm{~m}$ (0.65 mi.) to 3220 m (2.0 mi.) of U. S InterstatesI-24 and I-75 in Chattanooga, Tennessee. Roadroid runs were performed using a passenger car. A Samsung Galaxy S5 with Roadroid app (version 1. 7.5) 


\section{Civil Engineering Research Journal}

installed was mounted on the windshield of the car, since this study is being done on interstates, the data were collected at speeds ranging from $82 \mathrm{~km} / \mathrm{hr}$. (51mph.) to $92 \mathrm{~km} / \mathrm{hr}$. (56mph.).
If the car is moving above the specified speed ranges of Roadroid estimated IRI, the phone beeps and no measurements are recorded at that speed. Figure 1 shows photos captured by the app during the data collection process.
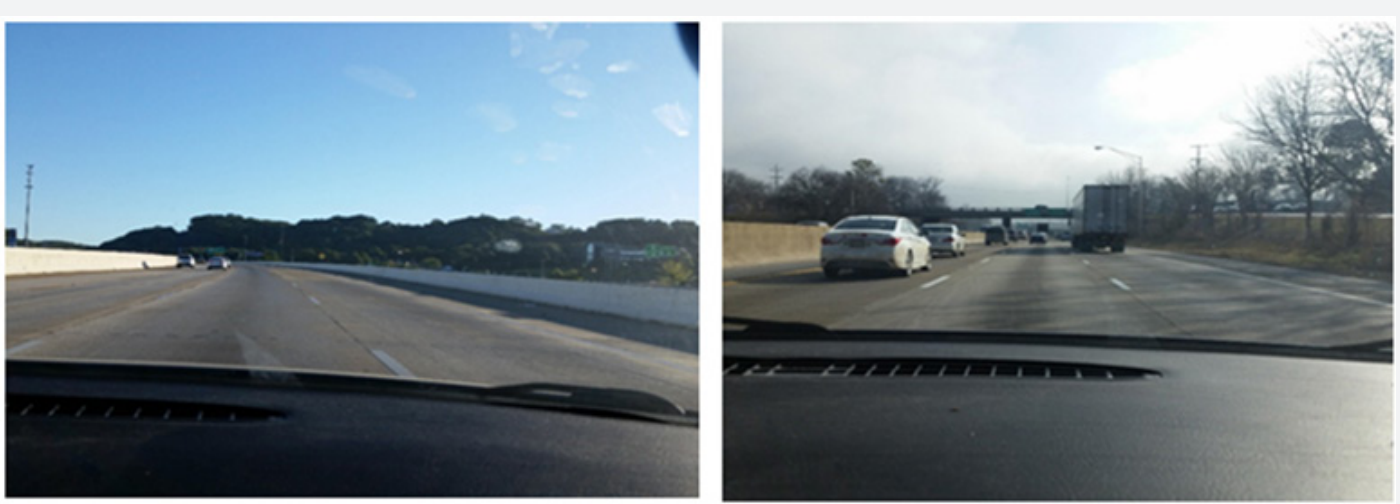

Figure 1: Sample photos captured by Roadroid app during data collection.

\section{Data Analysis}

The raw profile data collected by the standard profiler was analyzed using the Pro VAL software to obtain the IRI values. The standard profiler collects longitudinal profile of both wheel path (i.e. left and right wheel path) while Roadroid gives only the average IRI from both wheel path, therefore during the analysis of the profiler data, Mean Roughness Index (MRI) which is the average of the two (left and right) wheel path profiles was used.

Results Discussion

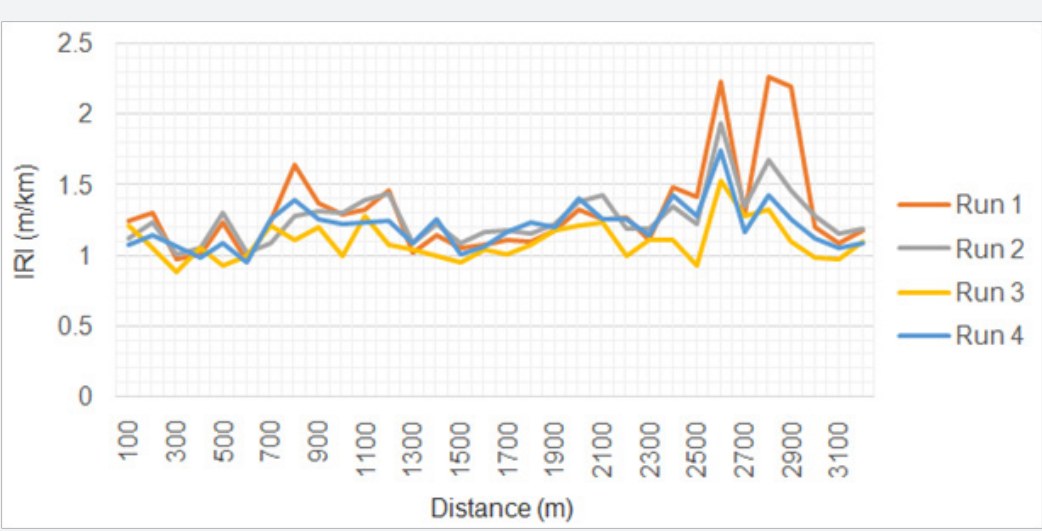

Figure 2: Repeatability of Roadroid IRI on I-75 North.

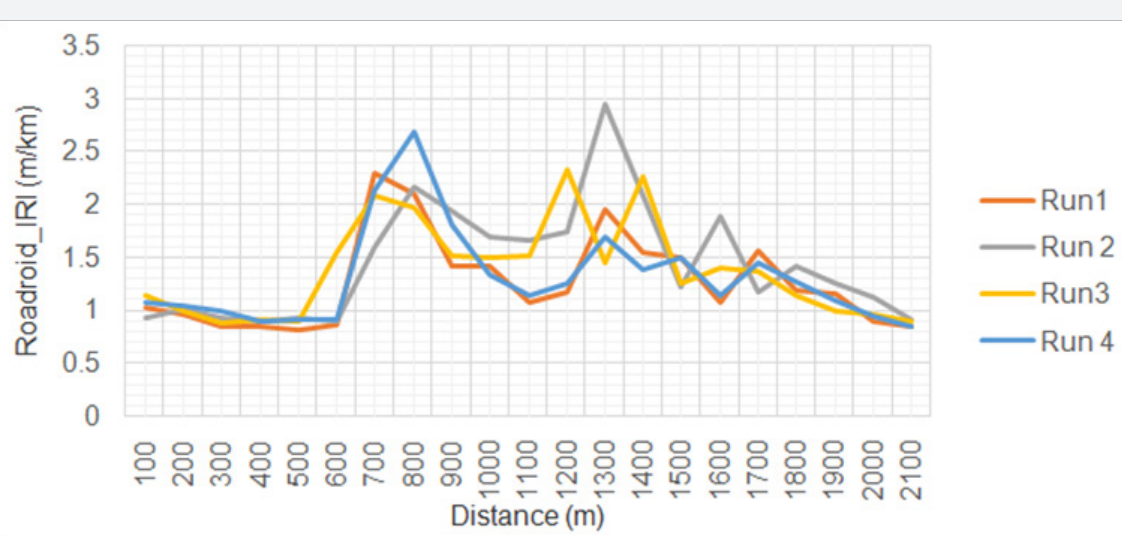

Figure 3: Repeatability of Roadroid IRI on I-24 West. 


\section{Civil Engineering Research Journal}

Figure 2 \& 3 show sample repeatability plots of Roadroid IRI measurements at every interval of $100 \mathrm{~m}(0.062 \mathrm{mi})$. All five runs are approximately in the same roughness range, despite deviations due to difference in lead in length during the start of the runs and vehicle tire wander.

The repeatability of IRI measurements can be increased by precisely matching the $100 \mathrm{~m}$ segments. The abrupt rise in the plots for instance up to IRI of $2.9 \mathrm{~m} / \mathrm{km}$ (Figure 3) indicates difference in elevations between the adjacent slabs, which is due to overcorrection during polyurethane injection. In depth assessment to evaluate the cause for high IRI is necessary at those specific points.

IRI measurements from the profiler are higher than those from all the Roadroid runs (Table 2). This is because the standard profiler has the ability of measuring the true longitudinal profile with accuracy of about $0.025 \mathrm{~mm}$. However, the Coefficient of Variation (CV) of the standard profiler measurements are higher than those of measurements from the Roadroid. This was influenced by high difference elevation among the adjacent slabs, which they are out of range for the Roadroid to record them.

Table 2: Inertial Profiler and Roadroid Roughness Measurements.

\begin{tabular}{|c|c|c|c|c|c|c|c|c|c|c|c|c|}
\hline \multirow{3}{*}{$\begin{array}{l}\text { Highway } \\
\text { Section ID }\end{array}$} & \multirow{3}{*}{ Length (m) } & \multirow{3}{*}{$\begin{array}{c}\text { lane } \\
\text { Treated }\end{array}$} & \multirow{2}{*}{\multicolumn{2}{|c|}{$\begin{array}{c}\text { Profiler MR1 }(\mathrm{m} / \mathrm{km}) \\
\text { Run } 1\end{array}$}} & \multicolumn{8}{|c|}{ Roadroid_Overall IR (m/km) } \\
\hline & & & & & \multicolumn{2}{|c|}{ Run 1} & \multicolumn{2}{|c|}{ Run 2} & \multicolumn{2}{|c|}{ Run 3} & \multicolumn{2}{|c|}{ Run 4} \\
\hline & & & Mean & CV (\%) & Mean & CV (\%) & Mean & CV (\%) & Mean & CV (\%) & Mean & CV (\%) \\
\hline 175 South & 3220 & 3 & 1.87 & 27.46 & 1.41 & 22.95 & 1.31 & 21.38 & 1.19 & 12.65 & 1.23 & 18.85 \\
\hline I 75 North & 3220 & 3 & 1.82 & 22. 19 & 1.32 & 25.36 & 1.27 & 14.98 & 1.1 & 12.56 & 1.2 & 13.15 \\
\hline I 24 East & 1050 & 3 & 2.85 & 31.01 & 1.51 & 17.54 & 1.26 & 15.4 & 1.3 & 15.76 & 1.29 & 25.83 \\
\hline 124 West & 2100 & 2 & 2.09 & 62.66 & 1.26 & 34.52 & 1.44 & 38.05 & 1.38 & 33.32 & 1.31 & 34.85 \\
\hline
\end{tabular}

A further statistical analysis was conducted by assuming a 95 percent confidence interval on all the four sections after dividing them at an interval of $100 \mathrm{~m}$. The difference of the measurements collected by the two instruments are statistically significant (i.e. all the p-values are less than 0.05).

Although standard profilers have a CV of less than $5 \%$ on a smooth road, the cost incurred to conduct a survey, made the app a preferred choice in monitoring the performance of the treated pavement sections.

Figure 4 \& 5 show a relationship between MRI from the standard profiler and Roadroid's IRI (run two) on I-75 North and I-24 West respectively. There is a linear correlation between the roughness readings from two tools, whereas most of the IRI readings from the standard profiler are above the identity line, indicating that Roadroid underestimates the IRI measurements.

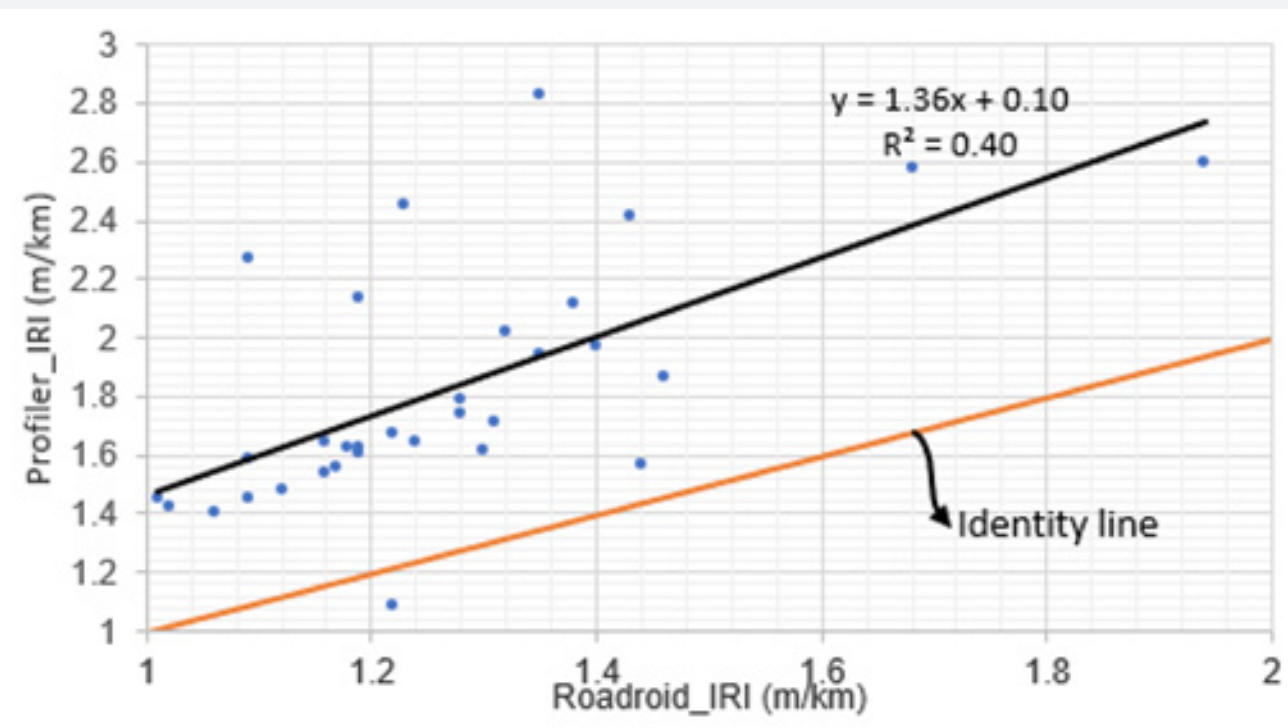

Figure 4: Relationship between profiler and Roadroid roughness on I-75 North 


\section{Civil Engineering Research Journal}

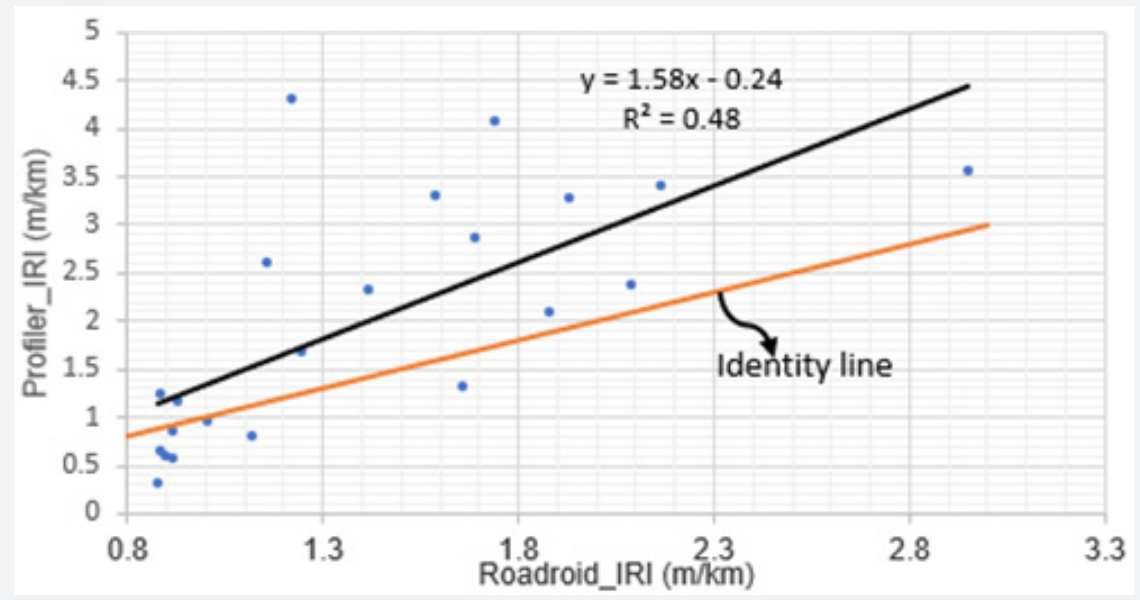

Figure 5: Relationship between profiler and Roadroid roughness on I-24 West.

\section{Conclusion}

IRI is a useful indicator on assessing the safety and comfortability experienced by road users as they travel on a road. It is also used to establish triggers and threshold levels that define appropriate timing of the treatment(s). In this project, estimated IRI from Roadroid is used to monitor and assess the performance of polyurethane treated sections to obtain objective values at less cost, rather than subjective that are likely to have human influences.

All four runs of Roadroid are repeatable with IRI values clustered within a narrow range but exhibiting minor deviations from each other most likely due to variation in lead in length, tire wander, and overcorrection of the treated concrete slabs. The CV of both the standard inertial profiler and the Roadroid app values varies with the roughness of the sections whereby rougher sections have higher variability as compared to sections with lesser roughness.

Roadroid underestimates the roughness measurements; and when its measurements compared to that of the standard profiler, the difference is statistically significant. However, road agencies with tight budgets can integrate Roadroid to their subjective pavement condition survey on low volume roads to obtain ride quality measurements with less human influence and make reasonable decision for pavement sections requiring treatment. For high volume roads, requiring high ride quality, using a standard profiler is necessary to evaluate if the desired quality has been attained.

\section{Acknowledgement}

This project is funded by TDOT to study the long-term performance of polyurethane material, particularly their extent on improving the ride quality of rigid pavements. Special thanks to TDOT Region 2 for working closely with the UTC team. Tami Johnson, a UTC undergraduate student for tirelessly working to obtain the Roadroid app and collecting initial roughness measurements.

\section{References}

1. Smith KD (2005) Concrete Pavement Rehabilitation and Preservation Treatments. F H Administration.

2. Vennapusa PK, White DJ (2015) Field assessment of a jointed concrete pavement foundation treated with injected polyurethane expandable foam. International Journal of Pavement Engineering 16(10): 906-918.

3. Sayers MW, Gillespie TD, Queiroz A (1986) The international road roughness experiment. Establishing correlation and a calibration standard for measurements.

4. Gillespie TD, Karamihas SM, Kohn, SD, Perera R (1999) Operational guidelines for longitudinal pavement profile measurement.

5. Sayers MW, Karamihas SM (1998) The little book of profiling. Transportation Research Institute, University of Michigan.

6. McGhee KH (2004) Automated pavement distress collection techniques: Transportation Research Board 334.

7. Haas R, Hudson WR, Zaniewski JP (1994) Modern pavement management.

8. Jones H, Forslof L (2014) Roadroid continuous road condition monitoring with smartphones. Paper presented at the Proceedings of the $5^{\text {th }}$ SARF/IRF Regional Conference, Pretoria, South Africa.

9. Morrow G (2006) Comparison of Roughness Measuring Instruments. (Masters Thesis), University of Auckland.

10. Gillespie TD (1980) Calibration of response-type road roughness measuring systems.

11. Woodstrom JH (1990) Measurements, Specifications, and Achievement of Smoothness for Pavement Construction: Transportation Research Board 167.

12. Islam S, Buttlar W, Aldunate R, Vavrik W (2014) Measurement of pavement roughness using android-based smartphone application. Transportation Research Record: Journal of the Transportation Research Board 2457: 30-38.

13. Hanson T, Cameron C, Hildebrand E (2014) Evaluation of low-cost consumer-level mobile phone technology for measuring international roughness index (IRI) values. Canadian Journal of Civil Engineering 41(9): 819-827.

14. Scholotjes M, Visser A, Bennett C (2014) Evaluation of a smartphone roughness meter. Proceedings of the $33^{\text {rd }}$ Southern African Transport Conference (SATC 2014).

15. Roadroid (2015) Road Roughness/IRI Survey Using Smartphones: Essentials for Professional Use. 


\section{Civil Engineering Research Journal} (C) This work is licensed under Creative

DOI: 10.19080/CERJ.2018.04.555645
Your next submission with Juniper Publishers will reach you the below assets

- Quality Editorial service

- Swift Peer Review

- Reprints availability

- E-prints Service

- Manuscript Podcast for convenient understanding

- Global attainment for your research

- Manuscript accessibility in different formats

( Pdf, E-pub, Full Text, Audio)

- Unceasing customer service

Track the below URL for one-step submission https://juniperpublishers.com/online-submission.php 